\title{
A new method using a polyglycolic acid monolayer patch to shield the mucosal defect after endoscopic submucosal dissection
}

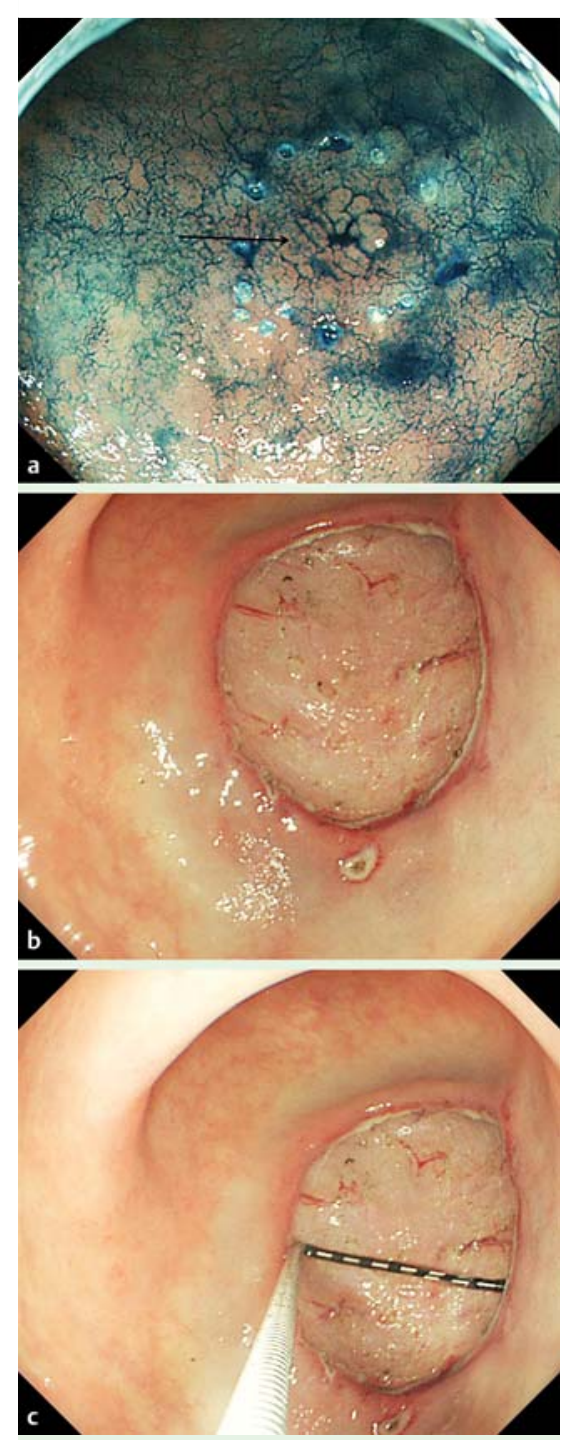

Fig. 1 Endoscopic views showing: a a cancer in gastric antrum (arrow); b the mucosal defect left following endoscopic submucosal dissection (ESD); $c$ the diameter of the mucosal defect being measured.

Recently, an endoscopic shielding method using polyglycolic acid (PGA) sheets (Neoviel, Gunze Co., Kyoto, Japan) and fibrin glue (Bolheal, Kaketsuken, Kumamoto, Japan) has been reported $[1,2]$. It is difficult to stretch a PGA sheet out as a monolayer during an endoscopic procedure because the sheets are very thin and their stickiness increases when wet. Therefore, in these previous reports, the mucosal defects have been covered with several
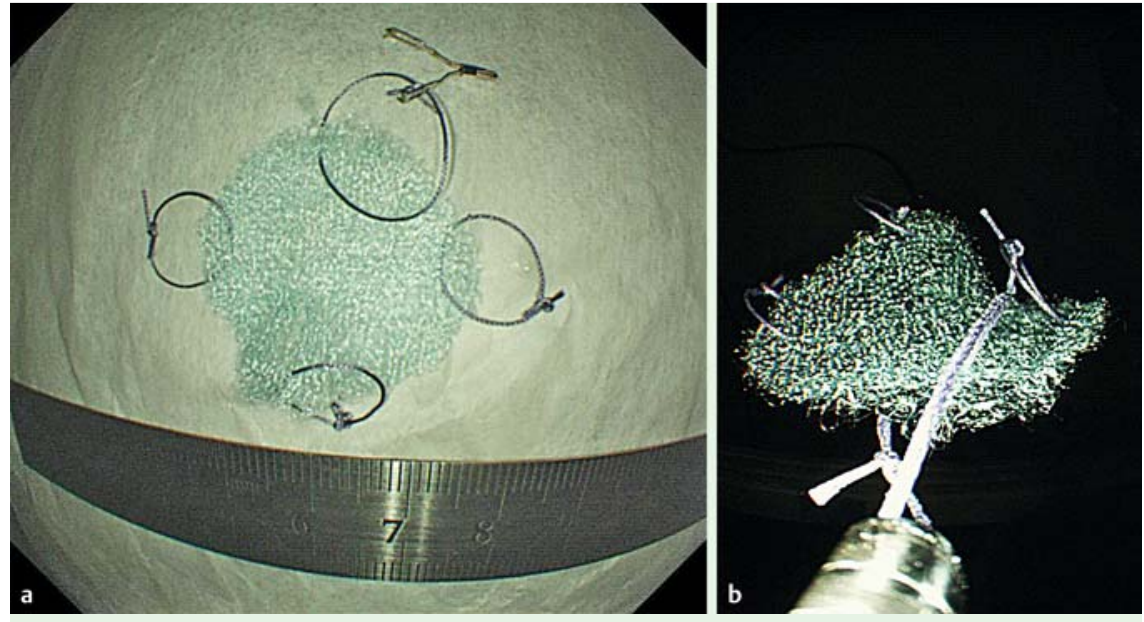

Fig. 2 Preparation of the polyglycolic acid (PGA) patch showing: a a single layer cut to the size of the defect with four loops of absorbable suture thread inserted through its margins at the four points of a cross; $\mathbf{b}$ the PGA patch being picked up by passing one of the loops through the hole of a Zeoclip that had been passed through the instrument channel of the endoscope.

strips of PGA [1,2]. Here, we report a new method that can be used to cover a gastric mucosal defect following endoscopic submucosal dissection (ESD) with just a monolayer of PGA.

In the case illustrated, the ESD was performed for an antral gastric cancer ( $\bullet$ Fig. 1a,b). The diameter of the resulting defect was measured ( $\bullet$ Fig.1c), and the endoscope was pulled out with the resected specimen. A patch that was shaped to fit the area of the defect was then cut from a sheet of PGA and four loops were created close to the edge of the material using absorbable suture thread at the four points of a cross ( Fig. $2 \mathbf{a}$ ). This prepared PGA patch was then picked up by passing one of the loops through the hole of a Zeoclip (Zeon Medical, Tokyo, Japan) that had been passed through the instrument channel of the endoscope (๑ Fig. 2b).

The endoscope with the PGA was re-inserted into the stomach and the clip was placed near to the margin of the mucosal defect ( $\bullet$ Fig.3a; $\bullet$ Video 1 ). The three remaining loops were then also fixed with clips around the margin of the defect ( $\bullet$ Fig.3b). Thereafter, the defect was completely covered with the PGA patch by adjusting the air content in the stomach and moving or stretching the PGA using a forceps and the additional clips

\section{Video 1}

The procedure for covering a mucosal defect following endoscopic submucosal dissection (ESD) with a monolayer patch of polyglycolic acid (PGA).

( $\mathrm{Fig} .3 \mathrm{c}$ ). Once the position was satisfactory, fibrin glue was injected to ensure adhesion of the patch to the defect.

We have already used this method in three other cases of gastric cancer ( $\bullet$ Fig.4). In all these cases, it was possible to completely cover the defect with a monolayer of PGA without any folds being formed. The mean additional time for this procedure was approximately 15 minutes.

\section{Endoscopy_UCTN_Code_TTT_1AO_2AC}

\section{Competing interests: None}

\section{Masakatsu Nakamura', Hisakazu Shiroeda', Tomomitsu Tahara², Tomoyuki Shibata ${ }^{2}$, Tomiyasu Arisawa ${ }^{1}$}

${ }^{1}$ Department of Gastroenterology, Kanazawa Medical University, Uchinada-machi, Japan

2 Department of Gastroenterology, Fujita Health University, Toyoake, Japan 

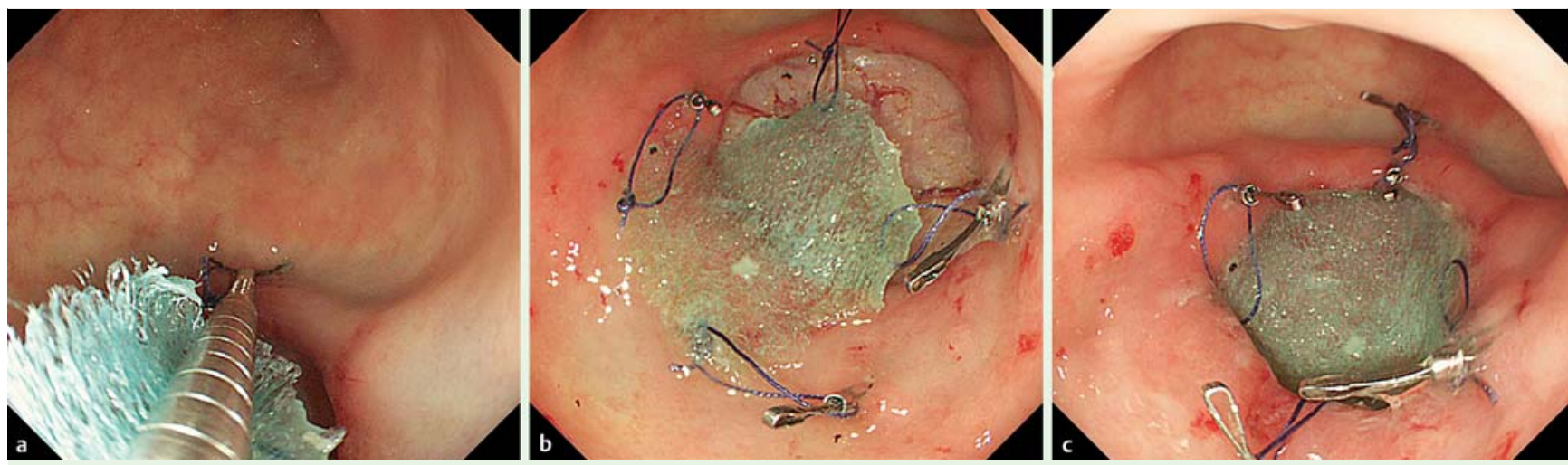

Fig. 3 Endoscopic views showing: a the clip being placed in the mucosa near to the margin of the mucosal defect; $\mathbf{b}$ all four loops having been fixed with clips around the mucosal defect; $\mathbf{c}$ the defect completely covered with the monolayer of PGA.

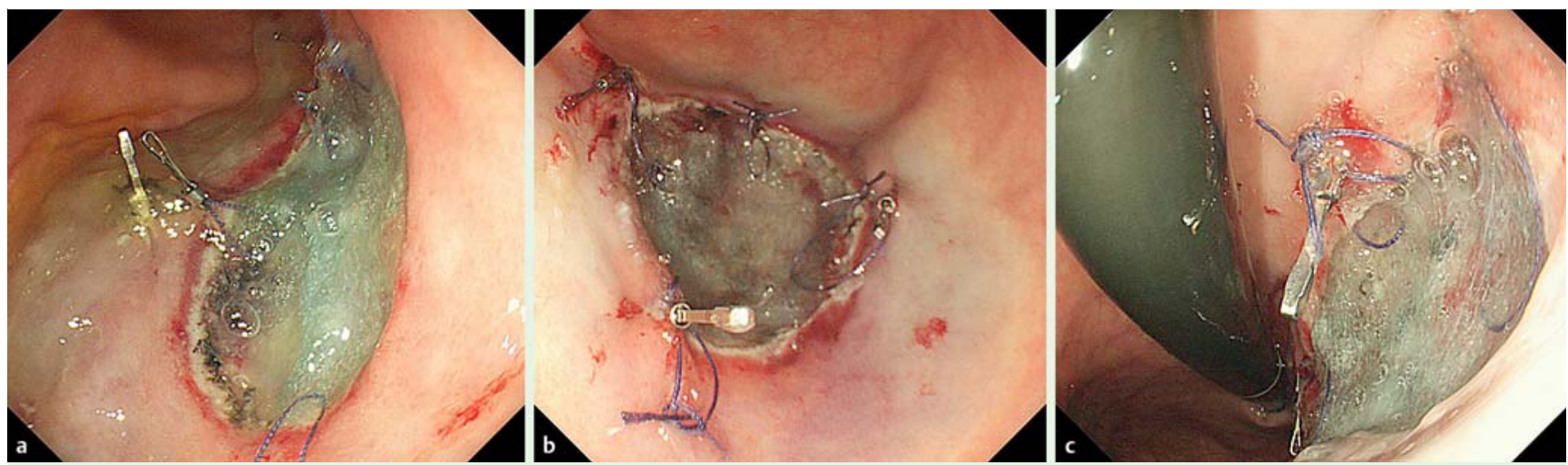

Fig. 4 Three further cases of gastric cancer in which this technique has been used showing lesions in: a the posterior wall of the lower body of the stomach; b the greater curvature of the lower body of the stomach; $\mathbf{c}$ the lesser curvature of the middle of the body of the stomach.

\section{References}

1 Takimoto K, Toyonaga T, Matsuyama K. Endoscopic tissue shielding to prevent delayed perforation associated with endoscopic mucosal dissection for duodenal neoplasm. Endoscopy 2012; 44: E414-E415

2 Tsuji Y, Ohata K, Gunji T et al. Endoscopic tissue shielding method with polyglycolic acid sheets and fibrin glue to cover wounds after colorectal endoscopic submucosal dissection (with video). Gastrointest Endosc 2014; 79: 151 - 155

\section{Bibliography}

DOI http://dx.doi.org/

10.1055/s-0034-1365105

Endoscopy 2014; 46: E176-E177

(c) Georg Thieme Verlag KG

Stuttgart · New York

ISSN 0013-726X
Corresponding author

Tomiyasu Arisawa, PhD, MD

Department of Gastroenterology

Kanazawa Medical University

1-1, Daigaku, Uchinada-machi

Ishikawa 920-0293

Japan

Fax: +81-76-2860892

tarisawa@kanazawa-med.ac.jp 